\title{
Do People with HIV Get the Dental Care They Need?
}

\section{Results of the HCSUS Study}

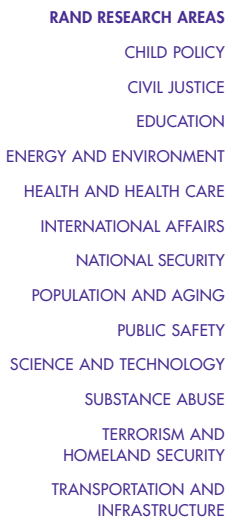

This product is part of the RAND Corporation research brief series. RAND research briefs present policy-oriented summaries of individual published, peer-reviewed documents or of a body of published work.

Corporate Headquarters 1776 Main Stree P.O. Box 2138 Santa Monica, California 90407-2138 Tel 310.393 .0411 Fax 310.393 .4818

(c) RAND 2005

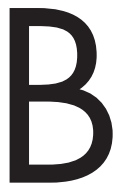

ecause of its effects on the immune system, HIV is a disease that affects every aspect of health. One of the earliest findings in the 20-year history of the HIV epidemic is that oral health was a frequent problem among HIV-infected individuals. In fact, opportunistic oral lesions and infections were often one of the first manifestations of HIV. Dental researchers have identified some 16 conditions that can occur in HIV-positive persons, some of them potentially life threatening. Thus, health experts agree that HIV-positive individuals (as well as those at increased risk for the disease) should see a dentist regularly. Yet anecdotes abound regarding dentists who refuse to treat people who are HIV-positive and HIV-positive individuals who avoid seeing a dentist out of fear of having to reveal their HIV status.

How likely is it that a person who is in regular medical care for HIV or full-blown AIDS will receive regular and appropriate dental care, and how common are the oral health conditions that have come to be seen as synonymous with HIV? Those are the questions that a group of researchers at RAND and the UCLA School of Dentistry has been trying to answer for the persons enrolled in the HIV Cost and Services Utilization Study (HCSUS). Their findings:

- In spite of the heightened risk of oral health problems in HIV-infected patients, a significant proportion of patients who receive regular medical care for HIV do not get the dental care they need.

- Many of those who report unmet needs for dental care-including Medicaid recipients

\section{Key findings}

- Many patients who receive regular medical care for HIV do not get the dental care they need.

- Many of those reporting unmet need for dental care-including Medicaid recipients whose state Medicaid program does not provide dental coverage-lack dental insurance.

- HIV-infected patients are more likely to get dental care when it's provided by the clinic where they get their medical care.

whose state Medicaid program does not provide dental coverage-lack dental insurance.

- HIV-infected patients who get their medical care at HIV clinics that provide dental care are more likely to get the dental care they need.

\section{People with HIV Don't Get Regular Dental Care}

In spite of the high rate of poor oral health among HIV-infected persons, 58 percent of HCSUS participants who completed the initial interview reported that they did not receive regular dental care. Regular dental care was defined as having received dental care in the six months preceding the initial interview. What characteristics differentiated those who did not receive regular dental care from those who did?

One characteristic that distinguished people who received regular dental care from those who did not was having a regular source of 


\section{What Is HCSUS?}

The HIV Cost and Services Utilization Study (HCSUS) is the first comprehensive U.S. survey of health care use among a nationally representative sample of HIV-positive persons who were in care for their HIV. The aims of HCSUS were to estimate the costs associated with HIV care; to identify barriers that affect access to HIV treatment as well as to other health care services; and to assess how HIV-positive status affects quality of life, productivity, and family life. Participants in HCSUS were interviewed several times over a 3-year period, making it possible to assess the effects of changes in HIV treatment.
Since it began in 1996, HCSUS has generated more than 70 original research publications on a wide variety of issues related to HIV infection. Highlights of the initial findings have been featured in several RAND Research Highlights (see back page). This Research Highlight reports findings from a series of questions posed during the initial interview regarding patients' use of dental care. The Highlight is one in a series summarizing HCSUS research. More information on HCSUS, including a list of publications, can be found at www.rand.org/health/hcsus. dental care: 65 percent of people who said that they had a regular dentist reported having seen the dentist, compared with only 12 percent of those who reported no regular source of dental care. Interestingly, 74 percent of those who said that their usual source of dental care was the dentist at their AIDS clinic had received care.

To assess all the personal characteristics that predict use of dental care, the researchers conducted a more complex type of analysis, known as a multivariate analysis, in which the effect of each factor is measured in groups of individuals who are identical in all ways except that one factor. The factors the researchers considered included race and ethnicity, gender, age, means of acquiring HIV, stage of disease (as measured by CD4 count, an indicator of the strength of a person's immune system), smoking history, educational achievement, employment status, income, and type of dental insurance, if any. Taking all possible factors into account in this way, the researchers found that being African American, having contracted HIV as a result of receiving blood products for hemophilia or blood transfusions, having less than a college education, and having no dental insurance decreased the likelihood of having seen a dentist in the previous six months. Interestingly, those who were unemployed were actually more likely to have seen a dentist. Figure 1 shows how these factors affect the likelihood of going without dental care. For example, participants with less than a high school (HS) education were two and a half times more likely not to have received regular dental care than those with a college degree.

\section{What About People Who Wanted to See a Dentist?}

People fail to get regular dental care for a variety of reasons, not the least of which is that they fail to perceive the need for such care. The researchers were interested in identifying barriers that HIV-infected persons might encounter in trying to get dental care. Thus, they needed to identify those individuals who, among those not receiving regular dental care, actually desired such care but believed they could not get it. Nearly 20 percent of the HCSUS participants reported having had a need for dental care in the previous six months that was not met. Figure 2 shows some factors that were associated with the perception of unmet need.

HIV-infected individuals with more-advanced disease are more likely to have oral health problems than those in the earlier stages of the disease, and so they are more likely to need dental care. Therefore, one might expect that those with the most-advanced disease would be more likely to report unmet needs for care than those in the early stages of HIV.

\section{Figure 1}

\section{Factors That Affect the Likelihood of Not Receiving Regular Care}

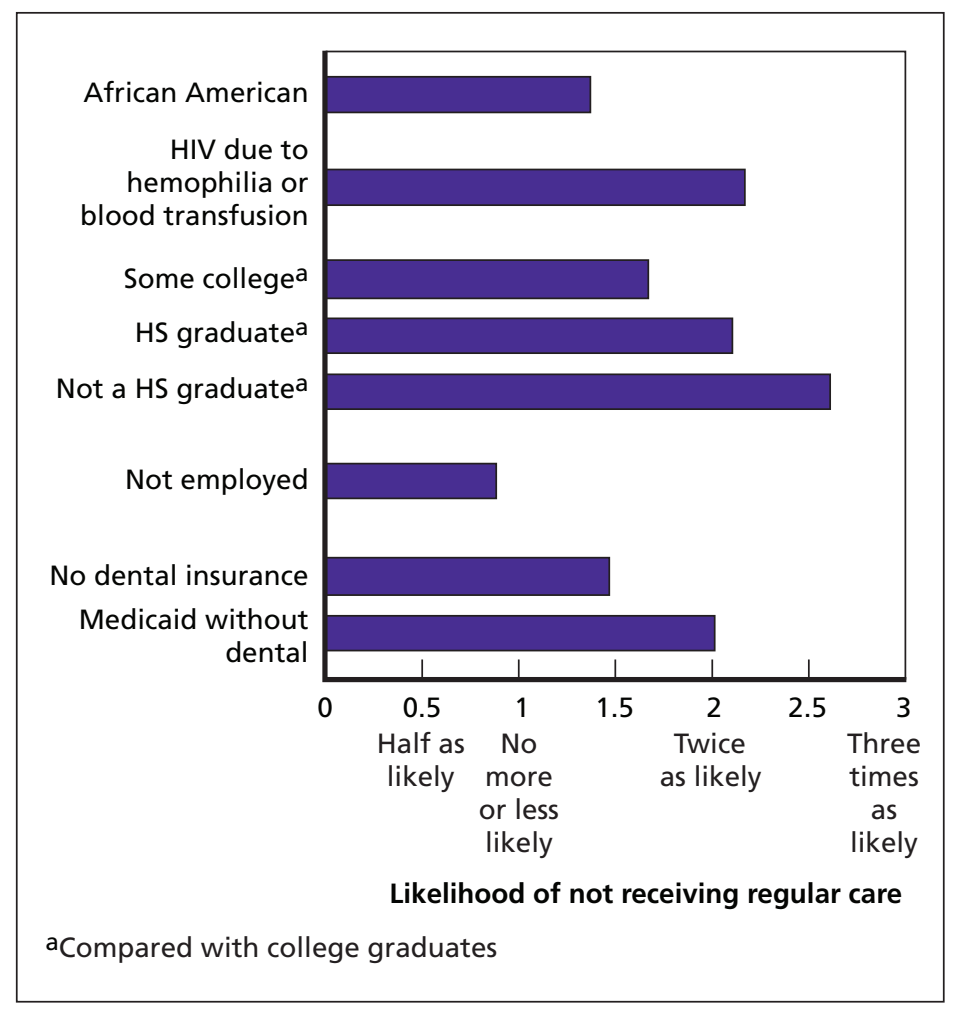


But the perception of unmet need for dental care was associated far more strongly with factors other than health status. These factors included age, usual source of dental care, educational attainment, income, employment status, smoking history, and dental insurance.

Figure 2 shows that participants over 50 were less likely to report unmet needs for dental care than younger participants. Those with less than a high school education were also more likely than those with more education to report an unmet need for dental care. Those whose yearly income was less than $\$ 10,000$ were more likely to report unmet needs for care than those with higher incomes. Those who reported not having a regular dentist were more likely than those with a regular dentist to have unmet needs for dental care, and smokers were less likely to report unmet needs for care than former smokers.

The perception of unmet need for dental care was greatest among participants who lacked dental insurance: Participants who reported unmet needs for dental care were more than twice as likely to lack dental insurance as those who did not. Some of these individuals were Medicaid recipients who lived in states in which Medicaid does not provide dental benefits; others were uninsured or had private health insurance, but no dental coverage.

\section{Figure 2}

\section{Factors That Affect the Likelihood of Perceiving an} Unmet Need for Dental Care

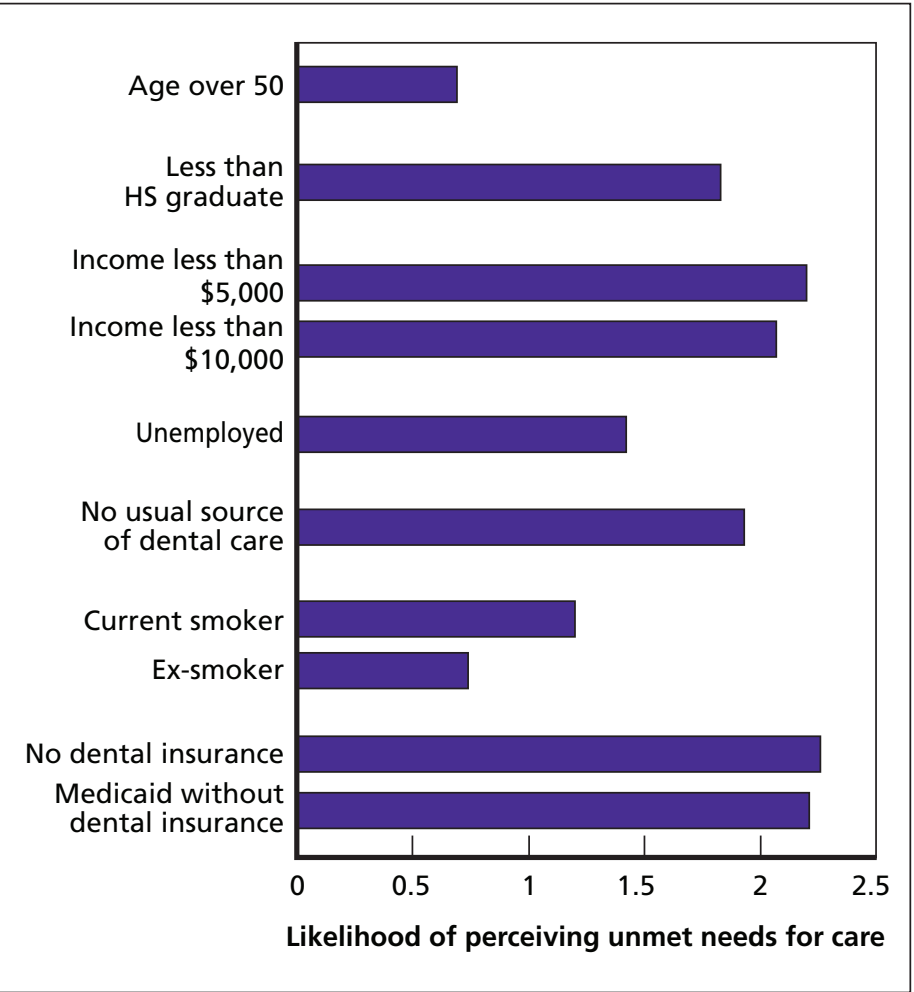

\section{Competing Health Care Needs Interfere with Seeking Dental Care}

As mentioned earlier, HIV-infected individuals with moreadvanced disease are more likely to need dental care than those in the earlier stages of the disease. But people in the advanced stages of HIV also tend to have more medical and mental health problems, which might make them less likely or able to seek needed dental care. The study confirmed that those who reported poorer physical and mental health were less likely to have visited a dentist. Thus, physical and mental health needs appear to compete with the need for oral health care for many individuals with advanced HIV. As a result, such individuals may not be receiving the oral health care they need.

The researchers also tried to determine whether the perception of unmet needs for dental care was more prevalent than the perception of unmet needs for medical care. What they found was that unmet dental needs were more than twice as common as unmet needs for medical care among the participants in our study. The uninsured and Medicaid recipients were the most likely to report unmet needs for both medical and dental care. It is important to keep in mind that HCSUS participants were selected from among a population of people receiving some medical care. Thus, the extent of unmet need for medical care is likely to be considerably higher among the general HIV-positive population than it is among HCSUS participants.

\section{Antiretroviral Therapy and Oral Health Care}

The use of highly active antiretroviral therapy (HAART), a cocktail of powerful drugs that reduces the severity of HIV, is responsible for the increased survival and quality of life of HIV-positive individuals witnessed throughout the 1990s. The researchers wondered if, by reducing the severity of HIV, HAART would lead to improvements in oral health as well as in physical health status. They found that people who began using HAART early in the course of their disease did show better oral health: Compared with those on HAART, patients receiving other treatments or not receiving antiviral treatments were far more likely to report having had one particular HIV-related opportunistic infection of the oral cavity, which appears in the form of white patches. Surprisingly, although HIV-infected African Americans are less likely to be receiving HAART therapy than white and Hispanic individuals, oral white patches were reported less frequently by African Americans. One possible reason is that African Americans who are HIV-infected are less likely to receive dental care than white persons who are HIV-infected; thus, they would be less likely to have a dentist bring the condition to their attention. 


\section{Addressing the Barriers to Seeking Oral Health Care}

HCSUS shows critical levels of unmet need for dental care in the HIV-infected population. Fortunately, in their attempt to characterize those most affected, the researchers have identified several straightforward policy modifications that have the potential to ameliorate the problem for many of those who report unmet needs for care.

First, access to affordable dental care could be increased if all state Medicaid programs began to provide dental coverage. Currently, only four states provide comprehensive dental coverage (11 states provide limited coverage, and another 20 states provide coverage for emergencies only). ${ }^{1}$

\footnotetext{
${ }^{1}$ Figures from Children's Dental Health Project, Preserving the Financial Safety Net by Protecting Medicaid and SCHIP Dental Benefits, September 2003. Available at www.cdhp.org.
}

Second, dental services could be offered at HIV clinics. Studies suggest that when patients whose illness or situation leaves them in a vulnerable condition can access all needed care in one convenient location-a kind of "one-stop shopping" for health care-access to and compliance with recommended care increase greatly. Access to dental care at HIV clinics would also eliminate the perceived stigma that hampers some HIV-infected individuals from seeking needed care.

The opportunistic infections that affect the oral health of HIV-infected individuals can, themselves, be life threatening. At the same time, they are often the earliest visible sign of HIV infection. These two factors underscore the need for regular dental care for those who are infected with HIV and those who are at increased risk. A few changes in dental coverage policy and accessibility of care can increase the likelihood that people affected by HIV will get the care they need. -

\section{This Highlight summarizes RAND Health research reported in the following publications:}

Access to HIV Care: Initial Results from the HIV Cost and Services Utilization Study, Santa Monica, Calif.: RAND Corporation, RB-4530, 2000. Available at www.rand.org/publications/RB/RB4530.

Caring for HIV Patients, Santa Monica, Calif.: RAND Corporation, RB-4525, 1999. Available at www.rand.org/publications/RB/RB4525.

Coulter ID, Heslin KC, Marcus M, Hays RD, Freed J, Der-Martirosian C, et al., "Associations of Self-Reported Oral Health with Physical and Mental Health in a Nationally Representative Sample of HIV Persons Receiving Medical Care," Quality of Life Research, Vol. 11, 2002, pp. $57-70$.

Coulter ID, Marcus M, Freed JR, Der-Martirosian C, Cunningham WE, Andersen RM, et al., "Use of Dental Care by HIV-Infected Medical Patients," Journal of Dental Research, Vol. 79, No. 6, 2000, pp. 1356-1361.

Dobalian A, Andersen RM, Stein JA, Hays RD, Cunningham WE, and Marcus M, "The Impact of HIV on Oral Health and Subsequent Use of Dental Services," Journal of Public Health Dentistry, Vol. 63, No. 2, 2003, pp. 78-85.

Heslin K, Cunningham WE, Marcus M, Coulter I, Freed J, Der-Martirosian C, et al., "A Comparison of Unmet Needs for Dental and Medical Care Among Persons with HIV Infection Receiving Care in the United States," Journal of Public Health Dentistry, Vol. 61, No. 1, 2001, pp. 14-21.

Marcus M, Freed JR, Coulter ID, Der-Martirosian C, Cunningham W, Andersen R, et al., "Perceived Unmet Need for Oral Treatment Among a National Population of HIV-Positive Medical Patients: Social and Clinical Correlates," American Journal of Public Health, Vol. 90 , No. 7, 2000, pp. 1059-1063.

Marcus M, Maida CA, Freed JR, Younai F, Coulter ID, Der-Martirosian C, et al., "Oral White Patches in a National Sample of Medical HIV Patients in the Era of HAART," Community Dentistry and Oral Epidemiology, Vol. 33, No. 2, April 2005, pp. 99-107.

A Portrait of the HIV+ Population in America: Initial Results from the HIV Cost and Services Utilization Study, Santa Monica, Calif.: RAND Corporation, RB-4523, 1999, www.rand.org/publications/RB/RB4523.

Abstracts of all RAND Health publications and full text of many research documents can be found on the RAND Health web site at www.rand.org/health. The RAND Corporation is a nonprofit research organization providing objective analysis and effective solutions that address the challenges facing the public and private sectors around the world. RAND's publications do not necessarily reflect the opinions of its research clients and sponsors. RAND ${ }^{\circledR}$ is a registered trademark.

RAND Offices Santa Monica - Washington - Pittsburgh • New York - Doha • Berlin - Cambridge • Leiden 
CHILD POLICY

CIVIL JUSTICE

EDUCATION

ENERGY AND ENVIRONMENT

HEALTH AND HEALTH CARE

INTERNATIONAL AFFAIRS

NATIONAL SECURITY

POPULATION AND AGING

PUBLIC SAFETY

SCIENCE AND TECHNOLOGY

SUBSTANCE ABUSE

TERRORISM AND HOMELAND SECURITY

TRANSPORTATION AND INFRASTRUCTURE
This PDF document was made available from www.rand.org as a public service of the RAND Corporation.

This product is part of the RAND Corporation research brief series. RAND research briefs present policy-oriented summaries of individual published, peerreviewed documents or of a body of published work.

The RAND Corporation is a nonprofit research organization providing objective analysis and effective solutions that address the challenges facing the public and private sectors around the world.

\section{Support RAND}

Browse Books \& Publications

Make a charitable contribution

For More Information

\author{
Visit RAND at www.rand.org \\ Explore RAND Health \\ View document details
}

Limited Electronic Distribution Rights

This document and trademark(s) contained herein are protected by law as indicated in a notice appearing later in this work. This electronic representation of RAND intellectual property is provided for noncommercial use only. Permission is required from RAND to reproduce, or reuse in another form, any of our research documents for commercial use. 